ARTICLE

https://doi.org/10.1038/s41467-019-09288-6

\title{
Overcoming the thermodynamic equilibrium of an isomerization reaction through oxidoreductive reactions for biotransformation
}

Jing-Jing Liu (1) 1, Guo-Chang Zhang (1) 1,2, Suryang Kwak¹,2, Eun Joong Oh¹,2, Eun Ju Yun 1,3, Kulika Chomvong ${ }^{4}$, Jamie H.D. Cate ${ }^{5}$ \& Yong-Su Jin ${ }^{1,2}$

Isomerases perform biotransformations without cofactors but often cause an undesirable mixture of substrate and product due to unfavorable thermodynamic equilibria. We demonstrate the feasibility of using an engineered yeast strain harboring oxidoreductase reactions to overcome the thermodynamic limit of an isomerization reaction. Specifically, a yeast strain capable of consuming lactose intracellularly is engineered to produce tagatose from lactose through three layers of manipulations. First, GAL1 coding for galactose kinase is deleted to eliminate galactose utilization. Second, heterologous xylose reductase (XR) and galactitol dehydrogenase (GDH) are introduced into the $\Delta$ gal1 strain. Third, the expression levels of $\mathrm{XR}$ and $\mathrm{GDH}$ are adjusted to maximize tagatose production. The resulting engineered yeast produces $37.69 \mathrm{~g} / \mathrm{L}$ of tagatose from lactose with a tagatose and galactose ratio of 9:1 in the reaction broth. These results suggest that in vivo oxidoreaductase reactions can be employed to replace isomerases in vitro for biotransformation.

\footnotetext{
${ }^{1}$ Carl R. Woese Institute for Genomic Biology, University of Illinois at Urbana-Champaign, Urbana, IL 61801, USA. ${ }^{2}$ Department of Food Science and Human Nutrition, University of Illinois at Urbana-Champaign, Urbana, IL 61801, USA. ${ }^{3}$ Department of Biotechnology, Graduate School, Korea University, Seoul 02841, South Korea. ${ }^{4}$ National Center for Genetic Engineering and Biotechnology (BIOTEC), 113 Thailand Science Park, Phahonyothin Road, Pathum Thani 12120, Thailand. ${ }^{5}$ Department of Molecular and Cell Biology, University of California, Berkeley, CA 94720, USA. Correspondence and requests for materials should be addressed to Y.-S.J. (email: ysjin@illinois.edu)
} 
$\mathrm{T}$ hermodynamic equilibrium where concentrations of substrates and products do not change with time is a fundamental limitation of enzymatic reactions catalyzed by isomerases $^{1}$. Biotransformations involving an isomerization reaction are therefore constrained by the underlying thermodynamic equilibrium, which often leads to an incomplete conversion of a substrate-generating a mixture of substrates and products. One typical example is the production of high-fructose corn syrup (HFCS) from glucose using glucose isomerase. Complete conversion of glucose into fructose is impossible due to the thermodynamic equilibrium of the reaction. Typically, a mixture of $42 \%$ fructose and $52 \%$ glucose is obtained by glucose isomerase ${ }^{2}$. In order to increase the concentration of fructose, separation and enrichment of fructose from the mixture are necessary.

Due to increasing concerns about excessive calories from added sugars in food, consumers seek a low-calorie alternative. Rare sugars such as tagatose and allulose, which possess a sweetness profile similar to sucrose but with much fewer calories, are promising low-calorie sweeteners in the food industry ${ }^{3-7}$. Rare sugar manufacturing usually involves isomerization reactions, resulting in a mixture of substrates and products after conversion ${ }^{8-11}$. As such, commercial production of the rare sugars requires additional separation steps. Manufacturing costs of rare sugars tend to be higher than HFCS and have impeded the wide use of rare sugars, even though they exhibit many benefits especially as potential anti-diabetic and anti-obesity medicines ${ }^{12,13}$. In particular, tagatose, a naturally occurring functional sweetener with $92 \%$ of the sweetness of sucrose but with substantially fewer calories $(1.5-2.5 \mathrm{kcal} / \mathrm{g} \text { vs. } 4 \mathrm{kcal} / \mathrm{g} \text { sucrose })^{14,15}$ has drawn great attention. Tagatose produced through isomerization of galactose is used as a food ingredient and attained GRAS (generally recognized as safe) status under the U.S. Food and Drug Administration (FDA). However, the manufacturing cost of tagatose by enzymatic isomerization remains high, partly due to the unfavorable thermodynamic equilibrium between galactose and tagatose ${ }^{16,17}$. Although the ratio of galactose and tagatose after enzymatic isomerization can be shifted to $4: 6$ by increasing reaction temperatures to $60^{\circ} \mathrm{C}$, the ratio of galactose and tagatose is around 7:3 under the temperatures, where L-arabinose isomerase (L-AI) is active and stable ${ }^{9,18}$. To obtain galactose as a substrate, enzymatic hydrolysis of lactose followed by a separation of glucose and galactose is required, and these additional steps can increase production costs. Moreover, the long-term and large-scale enzymatic conversion processes require maintenance costs, such as continued production of expensive purified enzymes. Therefore, enzyme-based tagatose production cannot compete with HFCS in the market despite its health benefits.

To bypass the above-mentioned bottlenecks, we devise a scalable and cost-effective tagatose production strategy based on direct yeast fermentation of lactose, an abundant sugar found in dairy by-products ${ }^{19}$. Specifically, we develope a whole-cell conversion process where lactose is transported into the cytosol of yeast, hydrolyzed, and converted into tagatose. In order to overcome the unfavorable equilibrium (7:3) by an isomerase reaction between galactose and tagatose at $30^{\circ} \mathrm{C}$, a two-step oxidoreductive pathway, consisting of aldose reductase ${ }^{20}$ and galactitol-2-dehydrogenase ${ }^{21}$, is introduced into an engineered Saccharomyces cerevisiae capable of assimilating lactose ${ }^{19}$. Additionally, to enhance the self-sustained production of tagatose in the lactose-assimilating yeast with the oxidoreductive pathway, galactose kinase (Gal1) is inactivated. As such, the resulting engineered yeast can take up lactose, produce glucose and galactose intracellularly. The intracellular glucose is used as the energy source for cell growth and maintenance, while galactose is simultaneously converted into tagatose. In this study, we demonstrate efficient tagatose production from lactose by the engineered yeast strain through a carbon-partition strategy whereby a consumed disaccharide is rerouted into different metabolic pathways-one for cell growth and maintenance, and another for targeted product generation. Interestingly, the production of tagatose directly from lactose by engineered yeast via the oxidoreductive pathway leads to a favorable conversion ratio (1:9) of galactose and tagatose, exceeding the thermodynamic equilibrium (7:3) by an isomerase reaction at $30^{\circ} \mathrm{C}$.

\section{Results}

A metabolic design to accumulate intracellular galactose. Previously, we constructed an engineered yeast strain (EJ2) to utilize cellobiose by introducing cellobiose transporter (CDT-1) and intracellular $\beta$-glucosidase (GH1-1) 22 . Notably, we found that the EJ2 strain also used lactose intracellularly ${ }^{19}$, suggesting that CDT1 can import lactose and GH1-1 can hydrolyze lactose as well as cellobiose. To accumulate intracellular galactose, we deleted the gene GAL1 coding for galactose kinase, which catalyzes the first step of the Leloir pathway responsible for galactose assimilation in yeast ${ }^{23}$. As expected, the GAL1-deleted strain (EJ2g) grew well on lactose, but accumulated galactose during lactose utilization (Fig. 1 and Supplementary Figure 1) in comparison with the parental strain (EJ2). No glucose accumulated in the culture medium with either strain. This result indicated that after lactose was hydrolyzed into glucose and galactose intracellularly, glucose supported cell growth of the EJ2g strain while galactose was not further metabolized. We therefore used the EJ2g strain as a host strain for intracellular conversion of galactose into tagatose.

Conversion of galactose into tagatose by oxidoreductases. To overcome the unfavorable equilibrium between galactose and tagatose by an isomerization reaction, we employed two oxidoreductases commonly used for the isomerization of aldose and ketose in yeast and fungi for the intracellular conversion of galactose into tagatose. As xylose reductase (XR) is known to reduce galactose into galactitol ${ }^{20}$, Scheffersomyces stipitis XYL1 coding for NADPH-linked XR was expressed in the EJ2g strain to produce galactitol from lactose. Expressing XR using a multicopy plasmid $(\mathrm{pRS} 42 \mathrm{~K})$ and a strong constitutive promoter $\left(\mathrm{P}_{T D H 3}\right)$ (EJ2g_pX) resulted in the production of $3.5 \mathrm{~g} / \mathrm{L}$ of galactitol during lactose fermentation (Fig. 2 and Supplementary Figure 2). To convert galactitol into tagatose, an $\mathrm{NAD}^{+}$-linked galactitol-2dehydrogenase (GDH) from Rhizobium leguminosarum ${ }^{21}$ was expressed in the galactitol-producing strain (EJ2g_pX) using a strong promoter $\left(\mathrm{P}_{T D H 3}\right)$ and a multicopy plasmid $(\mathrm{pRS} 42 \mathrm{H})$, resulting in the EJ2g_pXpG strain. The EJ2g_pXpG strain produced $11.43 \mathrm{~g} / \mathrm{L}$ of tagatose from $47.2 \mathrm{~g} / \mathrm{L}$ of lactose (Fig. 3 and Supplementary Figure 4). These data demonstrated that the two oxidoreductases (XR and GDH) functionally expressed in the engineered yeast (EJ2g_pXpG) and they enabled direct production of tagatose from lactose.

Fine-tuning of XR and GDH for enhanced tagatose production. While the engineered yeast EJ2g_pXpG produced tagatose from lactose, the observed yield $(0.242 \mathrm{~g}$ tagatose $/ \mathrm{g}$ lactose) was only half of the theoretical yield ( $0.526 \mathrm{~g}$ tagatose $/ \mathrm{g}$ lactose). We reasoned that the XR and GDH expression levels might need optimization to minimize the formation of by-products, such as galactose and galactitol (Fig. 3b, c), and maximize tagatose production. To elucidate the relationships between the galactose, galactitol, and tagatose production levels and the expression levels of XR and GDH, we constructed three more engineered strains with additional copies of XR and GDH via Cas9-based genome integration $^{24,25}$. The production of galactose, galactitol, and 

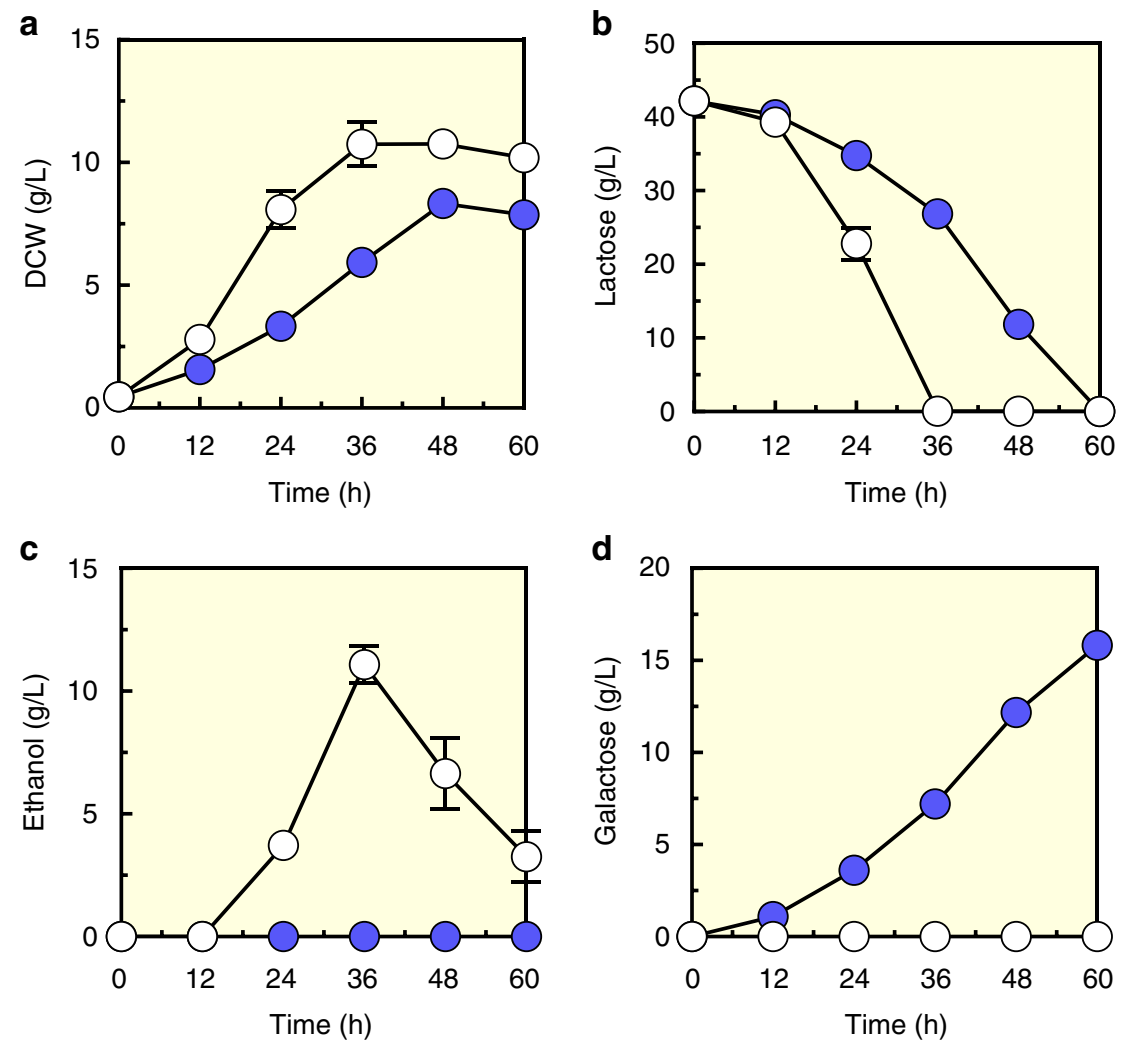

Fig. 1 Galactose accumulation in lactose-consuming strain EJ2 with GAL1 deletion (EJ2g). a Growth profile as shown by dry cell weight (DCW), b lactose consumption, $\mathbf{c}$ ethanol production, and $\mathbf{d}$ galactose production by the EJ2g strain (filled circles) as compared with the EJ2 control stain (open circles) under aerobic conditions. Data are presented as mean value and standard deviations of three independent biological replicates. Source data are provided as a Source Data file
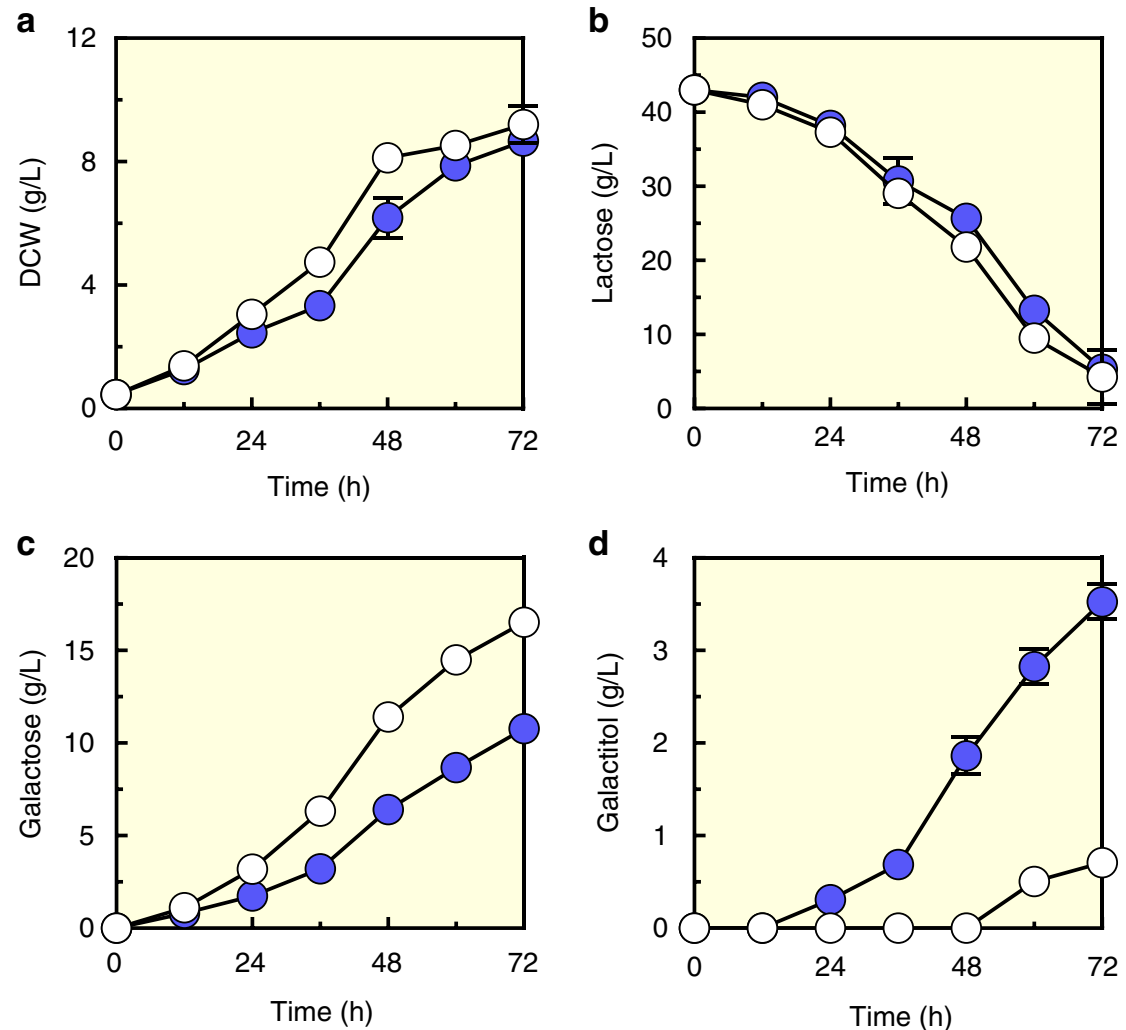

Fig. 2 Galactitol production after xylose reductase (XR) introduction into EJ2g. a Growth profile as shown by dry cell weight (DCW), b lactose consumption, $\mathbf{c}$ galactose production, and $\mathbf{d}$ galactitol produciton by the EJ2g strain with XR overexpression in the pRS42K plasmid (EJ2g_pX, filled circles), and EJ2g with empty plasmid pRS42K as control (open circles) on YP medium with $40 \mathrm{~g} / \mathrm{L}$ lactose under aerobic conditions. Data presented as mean values and standard deviations of three independent biological replicates. Source data are provided as a Source Data file 

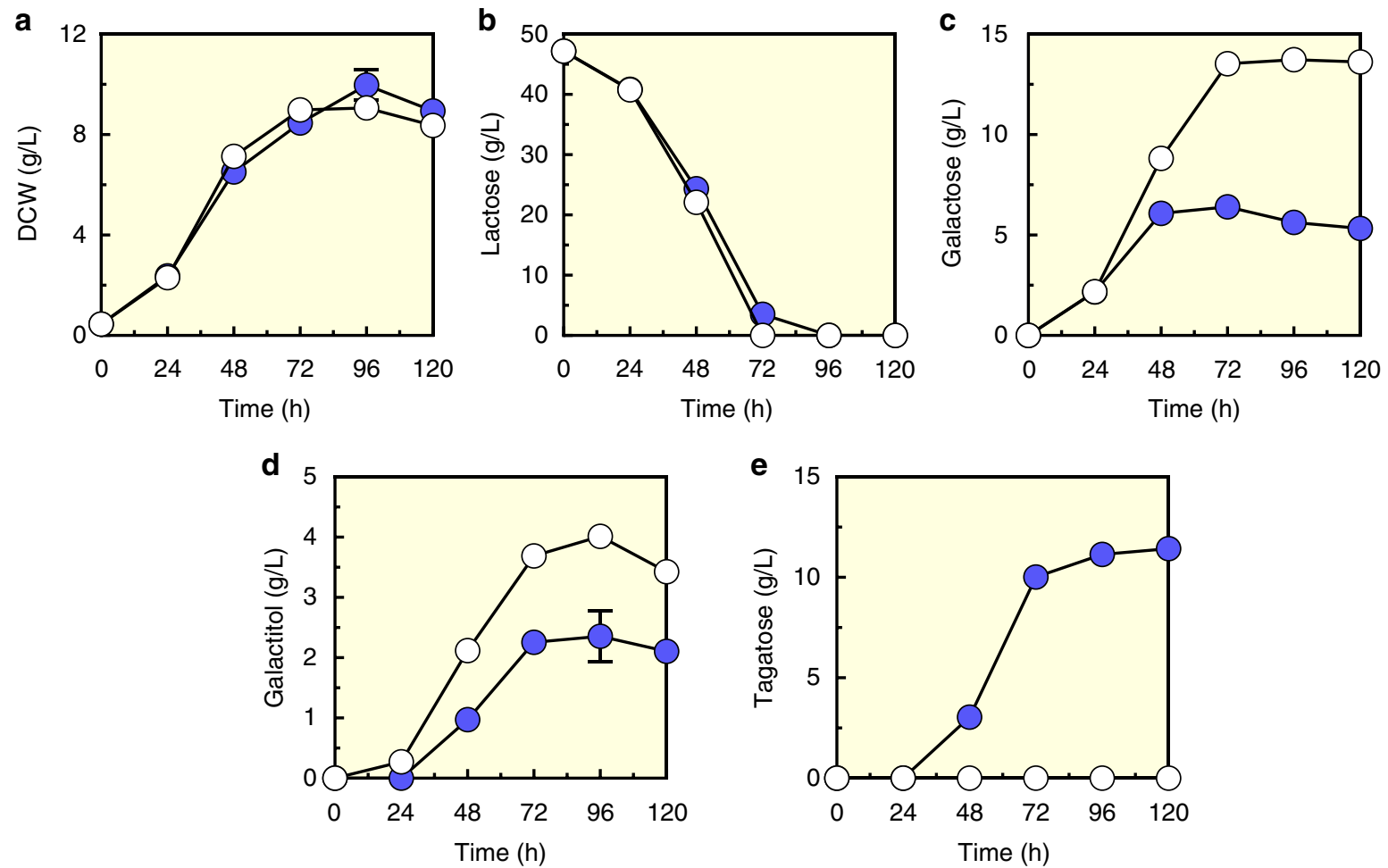

Fig. 3 Tagatose production after the introduction of galactitol-2-dehydrogenase (GDH). a Growth curve as shown by DCW, b lactose consumption, c galactose production, and $\mathbf{d}$ galactitol production, and $\mathbf{e}$ tagatose production by the EJ2g_pXpG strain (filled circles) and the EJ2g_pX strain with p42H empty plasmid as control (open circles) under aerobic condition. Data are presented as mean value and standard deviations of three independent biological replicates. Source data are provided as a Source Data file

\begin{tabular}{lll}
\hline \multicolumn{2}{l}{ Table 1 Engineered S. cerevisiae strains used in this study } \\
Strains & Description & Source \\
\hline EJ2 & Evolved strain of EJ1 (D452-2 leu2:LEU2 & 22 \\
& pRS405-gh1-1 ura3:URA3 pRS406-cdt-1) & \\
EJ2g & EJ2 $\Delta$ gal1 & This study \\
EJ2g_pX & EJ2g w/p42K-XR & This study \\
EJ2g_pXpG & EJ2g w/p42K-XR and p42H-GDH & This study \\
EJ2g_iX & EJ2g w/integrated pTDH3-XYL1-tTDH3 & This study \\
& in the CS6 locus & \\
EJ2g_iG & EJ2g w/integrated pTDH3-GDH-tCYC1 in & This study \\
& the CS8 locus & \\
EJ2g_iXiG & EJ2g w/integrated pTDH3-XYL1-tTDH3 & This study \\
& and pTDH3-GDH-tCYC1 & This study \\
EJ2g_iXiG_pX & EJ2g_iXiG w/p42K-XR & This study \\
EJ2g_iXiG_pG & EJ2g_iXiG w/p42H-GDH & This study \\
EJ2g_iXiG_pXpG & EJ2g_iXiG w/p42K-XR and p42H-GDH & This study \\
EJ2g_iX_pXpG & EJ2g_iX w/p42K-XR and p42H-GDH \\
EJ2g_iG_pXpG & EJ2g_iG w/p42K-XR and p42H-GDH & This study \\
\hline
\end{tabular}

tagatose from lactose by the four engineered yeast strains EJ2g_pXpG, EJ2g_iXiG_pX, EJ2g_iXiG_pG, and EJ2g_iXiG_pXpG (Table 1) was measured by HPLC (Fig. 4 and Supplementary Figure 5). Additional overexpression of XR in the EJ2g_iXiG_pX strain resulted in increased galactitol yield, but the yield of tagatose was unchanged. By contrast, additional overexpression of GDH in the EJ2g_iXiG_pG strain led to less galactitol accumulation and more tagatose production than the parental strain EJ2g_iXiG. The EJ2g_iXiG_pXpG strain with overexpression of both $\mathrm{XR}$ and GDH exhibited the highest tagatose and lowest galactitol yields (Fig. 4). These results suggested that XR and GDH expression levels played a critical role in manipulating the metabolic fluxes toward tagatose production. Using simple modifications in the expression levels of XR and $\mathrm{GDH}$, we increased the yield of tagatose from lactose by more than $20 \%$. The final galactose to tagatose ratio of EJ2g_iXiG_pXpG strain in the fermentation broth was 2:8.

Tagatose production in a bioreactor. To increase the titer of tagatose and to investigate the feasibility of large-scale production of tagatose from lactose, the EJ2g_iXiG_pXpG strain exhibiting the highest tagatose titer from a shake flask fermentation was tested in a bioreactor (Fig. 5 and Supplementary Figure 6). After $50 \mathrm{~g} / \mathrm{L}$ of lactose was consumed, additional feedings of lactose were conducted to maintain lactose concentrations around 10-20 $\mathrm{g} / \mathrm{L}$. After the fed-batch fermentation, $114.21 \mathrm{~g} / \mathrm{L}$ of lactose was consumed and the titers of tagatose, galactose, and galactitol were $37.69 \mathrm{~g} / \mathrm{L}, 4.41 \mathrm{~g} / \mathrm{L}$, and $8.46 \mathrm{~g} / \mathrm{L}$, respectively. As such, the conversion ratios of tagatose, galactose, and galactitol were $74.5 \%$, $8.7 \%$, and $16.7 \%$, respectively. The tagatose yield from lactose was $0.33 \mathrm{~g}$ tagatose $/ \mathrm{g}$ lactose, which is equivalent to $62.7 \%$ of a theoretical maximum ( $0.526 \mathrm{~g}$ tagatose/g lactose). Furthermore, the ratio of galactose to tagatose in the fermentation broth reached as high as 1:9 which is much higher than the ratio of 2:8 from the shake flask fermentation, substantially improving the $7: 3$ ratio achieved with the isomerase pathway at $30^{\circ} \mathrm{C}$. The concentration of galactose reached a steady-state level during the lactose feeding process (Fig. 5), suggesting that galactose can be converted efficiently into galactitol and tagatose with the lactose feeding rates used in this experiment. The galactitol yield in the fed-batch fermentation $(0.074 \mathrm{~g}$ galactitol $/ \mathrm{g}$ lactose vs. $0.035 \mathrm{~g}$ galactitol $/ \mathrm{g}$ lactose) was higher than that in the shake flask fermentation, suggesting that GDH activity and supply of $\mathrm{NAD}^{+}$might need to be further optimized to cope with higher metabolic fluxes from lactose and galactose. 


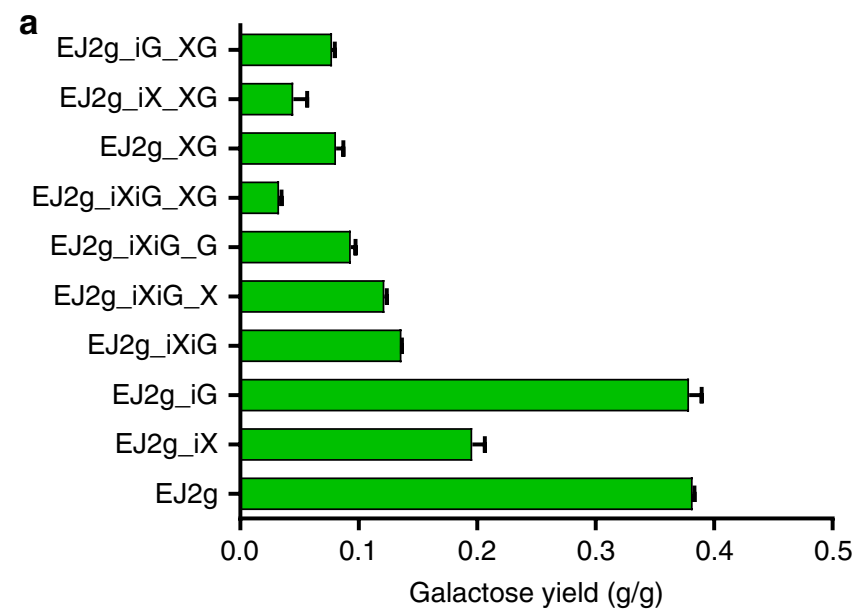

b
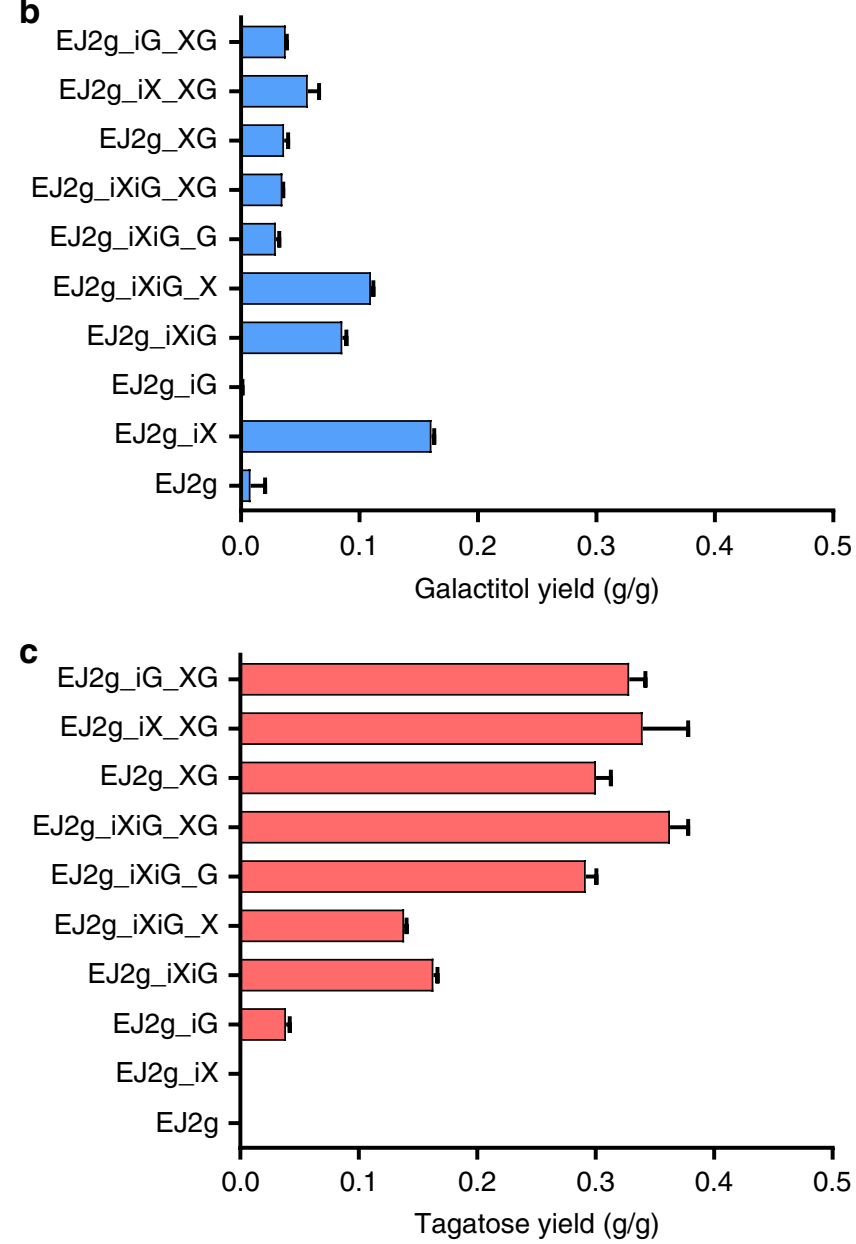

Fig. 4 Improved production of tagatose by increasing the XR and GDH gene copy numbers. a Galactose yield, $\mathbf{b}$ galactitol yield, and $\mathbf{c}$ tagatose yield. Data are presented as mean value and standard deviations of three independent biological replicates. Source data are provided as a Source Data file

\section{Discussion}

In this study, we bypassed the thermodynamic equilibrium of the isomerase reaction by using an oxidoreductive pathway to achieve the one-pot biosynthesis (Fig. 6). Tagatose production was selected as a demonstration system as its production was shown previously to be limited by the thermodynamic equilibrium ${ }^{8,9,18,26-28}$.
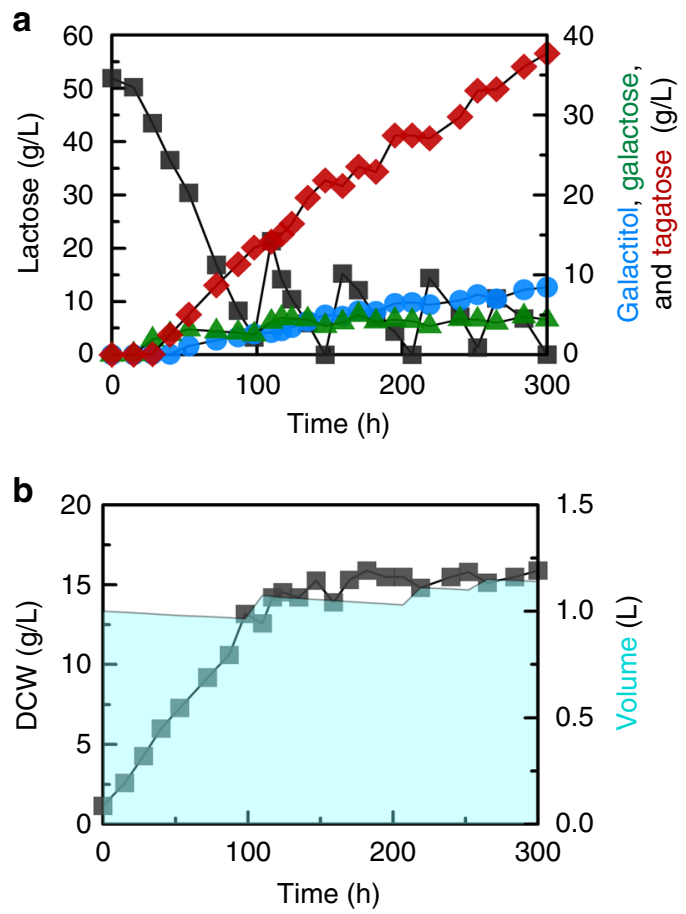

Fig. 5 Tagatose production by the EJ2g_iXiG_pXpG strain in a $2 \mathrm{~L}$ bioreactor. a Profiles of lactose, galactose, galactitol, and tagatose concentrations, and $\mathbf{b}$ the growth of the EJ2g_iXiG_pXpG strain and the volume changes of bioreactor during the fed-batch fermentation. Source data are provided as a Source Data file

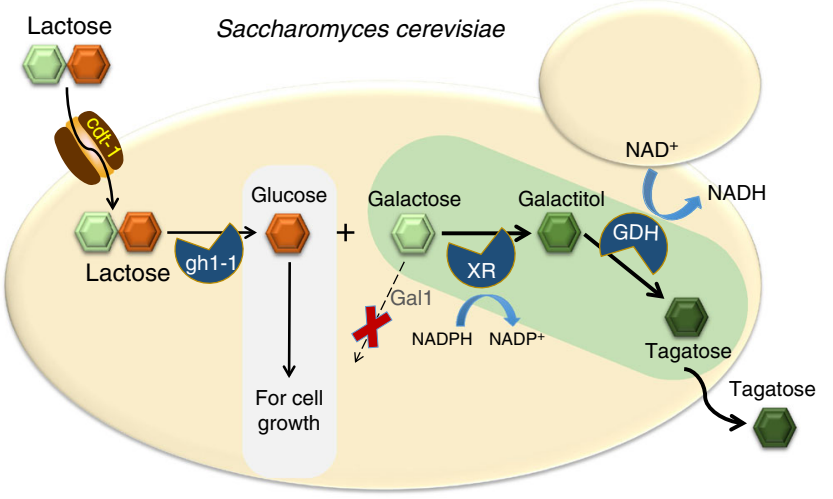

Fig. 6 Schematic diagram of the production of tagatose from lactose in engineered S. cerevisiae. Heterologous expression of cdt-1 (cellobiose transporter), gh1-1 ( $\beta$-glucosidase), XR (xylose reductase), GDH (galactitol2-dehydrogeanse), and deletion of Gal1 (galactose kinase) were combined in S. cerevisiae to produce tagatose directly from lactose

Interconversion between aldose and ketose forms of sugars are critical for their assimilation by microorganisms. Both single-step isomerization and two-step oxidoreductive reactions have evolved to catalyze these interconversions. For instance, xylose can be converted into xylulose via either xylose isomerase (XI) or the combined action of xylose reductase (XR) and xylitol dehydrogenase $(\mathrm{XDH})^{29}$. The XI reaction is often associated with anaerobic or microaerophilic bacteria ${ }^{30,31}$, due to the fact that the isomerase enzyme does not require cofactors and can carry out bioconversions under oxygen-limited and anaerobic conditions. Isomerase reactions have been adopted for industrial production of various value-added chemicals, but face two fundamental drawbacks in their use. First, the inherent thermodynamic 
equilibrium between the substrate and product leads to low conversion yields and creates difficulties for downstream products separation and purification. Second, the scale-up cost sharply increases, because the amount of enzyme required is directly proportional to the proposed reaction scale. For example, rare sugars such as tagatose and allulose are currently produced by enzymatic reactions followed by complicated separation processes. As a result, the production costs of rare sugars are significantly higher than HFCS, which does not require additional separation. Due to this high production cost, introduction of rare sugars into foods and beverages has been hampered in spite of potential benefits of rare sugars ${ }^{4}$.

Unlike the isomerase reaction, the oxidoreductive reaction consists of two steps (an oxidation and a reduction) and requires cofactors. Though seemingly redundant, this mechanism prevents the undesired reverse reaction through thermodynamic coupling to cofactor equilibria. Therefore, we used the oxidoreductive pathway instead of the isomerase pathway, to achieve more complete conversion of galactose into tagatose product than the isomerization. To implement the oxidoreductive pathway for tagatose production, in vivo bioconversion is more suitable than in vitro enzymatic conversion, considering the need for redox cofactors. Simultaneously, an efficient self-sustained bioconversion system is advantageous when considering scale-up costs. While direct and efficient conversion of lactose into tagatose by the oxidoreductive pathway has been demonstrated in this study, the secretion of galactose and galactitol during the conversion needs to be addressed in order to reach the theoretical maximum yield. We speculate that the secretion of galactose and galactitol might be facilitated by endogenous hexose and sugar alcohol transporter in yeast. Specifically, Gal2 (galactose permease) might be responsible for the secretion and re-assimilation of galactose, and Fps1 (aquaglyceroporin), which have been reported to transport xylitol ${ }^{32}$, might be involved in the secretion of galactitol. From the structural similarity between galactitol and xylitol, the deletion of FPS1 in a tagatose-producing strain might reduce production of galactitol. As Gal2 might be involved in both secretion and re-assimulation of galactose, upregulation of GAL2 might increase the secretion and re-assimilation of galactose, and deletion might reduce the secretion and re-assimilation of galactose. As such, the effects of GAL2 perturbation would likely be mixed or compromised. Genetic perturbations of endogenous sugar and sugar alcohol transporters including FPS1 and GAL2 can be conducted in the future to further improve the conversion yield of tagatose from lactose. In addition to galactitol accumulation as a by-product, the productivity and titer of tagatose from lactose by our engineered yeast need to be further improved. While the productivity $(0.126 \mathrm{~g} / \mathrm{L} \cdot \mathrm{h})$ and titer $(37.69 \mathrm{~g} / \mathrm{L})$ of tagatose production from lactose by our engineered yeast are comparable with the reported productivities $(0.103-0.896 \mathrm{~g} / \mathrm{L} \cdot \mathrm{h})$ and titers $(14.8-21.5 \mathrm{~g} / \mathrm{L})$ by enzyme-based tagatose production from lactose ${ }^{18,33}$, they are much lower than the productivity $(7.9 \mathrm{~g} / \mathrm{L} \cdot \mathrm{h})$ and titer $(158 \mathrm{~g} / \mathrm{L})$ by enzyme-based tagatose production from galactose ${ }^{5}$. Continuous fermentation with cellrecycling might be a possible approach to improve the productivity of tagataose production from lactose by our engineered yeast.

We also designed a carbon partition strategy that utilizes lactose, a disaccharide of galactose, and glucose, as an initial substrate for simultaneous tagatose production and glucose catabolism. Specifically, we intentionally shut down the native galactose utilization pathway of $S$. cerevisiae and redirected galactose toward tagatose through the introduced oxidoreductive pathway. Meanwhile, glucose was consumed to sustain the engineered yeast via its native pathway. Because the two monosaccharides were released intracellularly, the glucose repression on galactose uptake was bypassed and thus allowed for simultaneous utilization ${ }^{34,35}$. Hydrolysis of lactose into glucose and galactose, and the subsequent conversion of galactose to tagatose, were integrated and self-sustained, which dramatically reduced processing costs. It is worth noting that the carbon-partition strategy is not only limited to tagatose production but can also be applied in other bioconversion systems. When consuming disaccharides, we can opt to turn off the native catabolic pathway of one of the monosaccharide moieties and reprogram this monosaccharide toward our target chemicals, while leaving another moiety for cell growth and maintenance. If it is simplier to produce the target chemical using glucose rather than galactose as a substrate, we can turn off the glucose pathway by disrupting the hexose kinases (encoded by HXK1 and HXK2) and glucose kinase (encoded by GLK1). We can then introduce a target oxidoreductive pathway to allow glucose rerouting to the target chemicals, while maintaining the native galactose pathway for cell maintenance. Apart from lactose, other disaccharides such as sucrose are also cheap and abundant. Many other engineered yeast strains rapidly consume these disaccharides as well. Thus, we can produce target chemicals from these disaccharides as needed through the carbon-partition strategy.

In this study, our strategy reduces the production cost at every step, as compared with existing industrial tagatose production systems. First, the traditional process requires that the majority of galactose is made from enzymatic hydrolysis of lactose, followed by the separation of glucose and galactose. Therefore, direct consumption of lactose by our engineered yeast strain can significantly reduce the enzyme ( $\beta$-galactosidase) and separation costs. In addition, lactose is readily obtained from whey, an abundant waste product from cheese and Greek yogurt production. Next, the in vivo oxidoreductive conversion of galactose into tagatose eliminated the cost of purified or immobilized $\mathrm{L}$-arabinose isomerase. Unlike the traditional process, where enzyme cost increases proportionally with the scale of operation, the engineered yeast replicates itself continuously regardless of the reaction scale. Most importantly, the oxidoreductive reaction allows near-complete conversion of galactose into tagatose. The small amounts of galactose in the fermentation broth can be completely removed by adding regular yeast that consumes galactose to simplify the downstream separation process. Therefore, our strategy not only maximizes substrate value, but also simplifies product separation and purification, resulting in an overall decrease in production costs. In future studies, we intend to optimize the oxidoreductive galactose-tagatose pathway, to increase tagatose yields, to achieve complete conversion of galactose into tagatose, and to further minimize the separation and purification cost. In summary, we envision the carbonpartition strategy applicable for value-added chemical productions from disaccharides by engineered microorganisms. Our scheme allows for the disaccharides to be utilized intracellularly, and its monosaccharide products allotted for maintenance and production separately, but simultaneously.

\section{Methods}

Strains and media. Escherichia coli Top10 (Invitrogen, Grand Island, NY) was used for the construction and propagation of plasmids. E. coli was grown in the Luria-Bertani medium $(5 \mathrm{~g} / \mathrm{L}$ yeast extract, $10 \mathrm{~g} / \mathrm{L}$ tryptone, $10 \mathrm{~g} / \mathrm{L} \mathrm{NaCl}, \mathrm{pH} 7.0)$ at $37^{\circ} \mathrm{C}$, and ampicillin $(100 \mu \mathrm{g} / \mathrm{mL})$ was added for selection when required. Yeast strains were grown on the YPD medium $(10 \mathrm{~g} / \mathrm{L}$ yeast extract, $20 \mathrm{~g} / \mathrm{L}$ peptone, and $20 \mathrm{~g} / \mathrm{L}$ glucose) at $30^{\circ} \mathrm{C}$. Yeast strains transformed with plasmids containing antibiotic markers were propagated on YPD plates supplemented with the corresponding antibiotics, such as hygromycin B $(300 \mu \mathrm{g} / \mathrm{mL})$ and/or geneticin G418 $(300 \mu \mathrm{g} / \mathrm{mL})$.

Plasmids and strain construction. The guide RNA plasmid p42H-gGAL1 (Supplementary Table 3) was amplified from gRNA-ura-HYB ${ }^{25,36}$ used as a template by 
using primer pair Gall-gU and Gall-gD (Supplementary Table 1) carrying a $20 \mathrm{bp}$ PAM sequence for GAL1 deletion. Donor DNA was amplified using primers Gal1Donor-U and Gal1-Donor-D (Supplementary Table 1). GAL1 was deleted in the EJ2 strain ${ }^{22}$ using CRISPR-Cas9 technology ${ }^{25}$. Plasmid CAS9-NAT (Supplementary Table 3) was transformed into the EJ2 strain followed by guide RNA plasmid (p42H-gGAL1) and donor DNA transformation. The transformants were diagnosed for GAL1 deletion using primers Gal1-CK-U and Gall-CK-D (Supplementary Table 1). Plasmid pYS10 ${ }^{37}$ was digested by SacI and XhoI, and the pTDH3-XYL1-tTDH3 cassette was ligated with the same enzyme-digested pRS42K to construct p42K-XR (Supplementary Table 3). The pGPD-tCYC1 cassette from plasmid p426-pGPD ${ }^{38}$ was double digested by SacI and KpnI and ligated with SacI and $K p n I$ digested pRS42 $\mathrm{H}^{39}$, forming plasmid p42H-pGPD (Supplementary Table 3). The gene fragment of GDH was synthesized as gBlocks (IDT Inc, Skokie, IL) and blunt-ligated with plasmid p42H-pGPD to generate plasmid p42H-GDH. In order to integrate target genes into the genome of strain EJ2g for stable expression, CS6 and CS8 loci were used. The CS6 and CS8 sites are selected intergenic regions for integration of expression cassettes via Cas9-based genome editing. The CS6 site is located between YGR190C and tW(CCA)G2 in Chromosome VII, and the CS8 is located between YPR014C and YPR015C in Chromosome XVI. Targeting guide RNA sequences for CS6 and CS8 are listed in Supplementary Table 2. Detailed sequence information of the CS6 and CS8 sites are provided in Supplementary Data 1 and Supplementary Data 2. Based on the targeting sequences, guide RNA-expressing plasmids (p42K-CS8 and p42K-CS6) were constructed by reverse PCR of a pRS42K plasmid-containing guide RNA sequence using primer pairs gCS6-U/gCS6-D and gCS8-U/gCS8-D, respectively (Supplementary Table 1$)^{40}$.

For genomic integration of XYL1 into the EJ2g strain, the plasmid p42K-XR was amplified using a primer pair of CS6-IU and CS6-ID as donor DNAs for CRISPR-Cas9-based integration. The transformants with CS6-XR integration were confirmed by PCR using primers CS6-CKU and CS6-CKD, and was designated as the EJ2g_iX strain (Table 1). Similarly, CS8-GDH was integrated into the EJ2g_iX strains with a donor DNA amplified from plasmid $\mathrm{p} 42 \mathrm{H}-\mathrm{GDH}$ using primers CS8IU and CS8-ID. The transformants were confirmed by PCR using primers CS8CKU and CS8-CKD, and the engineered strain with CS6-XR and CS8-GDH integration was named as the EJ2g_iXiG strain (Table 1).

Yeast culture and fermentation conditions. The lactose fermentation was started by inoculating the overnight yeast pre-culture $(5 \mathrm{~mL}$ of the YP medium containing $20 \mathrm{~g} / \mathrm{L}$ of cellobiose) into $20 \mathrm{~mL}$ YPL (the YP medium containing $40-50 \mathrm{~g} / \mathrm{L}$ of lactose) in a $125 \mathrm{~mL}$ Erlenmeyer flask to obtain an initial optical density at $600 \mathrm{~nm}$ $\left(\mathrm{OD}_{600}\right)=1.0$ then incubated at $30^{\circ} \mathrm{C}$ with shaking (orbit of $2.5 \mathrm{~cm}$ ). The engineered yeast strain was precultured in $100 \mathrm{~mL}$ of the YP medium containing $20 \mathrm{~g} / \mathrm{L}$ of lactose with hygromycin $(300 \mu \mathrm{g} / \mathrm{mL})$ and $\mathrm{G} 418(300 \mu \mathrm{g} / \mathrm{mL})$ at $30^{\circ} \mathrm{C}$ with shaking (orbit of $2.5 \mathrm{~cm}$ ) with baffled flasks for 2 days. Cells at stationary phase were harvested at $15,294 \times g$ for $2 \mathrm{~min}$, and suspended in sterilized water before inoculation. Fed-batch culture was conducted in BioFlo \& CelliGen (R) 310 fermenter (New Brunswick Scientific-Eppendorf) with the YP medium containing $50 \mathrm{~g} / \mathrm{L}$ of lactose with $300 \mu \mathrm{g} / \mathrm{mL}$ of hygromycin and $300 \mu \mathrm{g} / \mathrm{mL}$ of G418 at $30^{\circ} \mathrm{C}$. The initial cell density was $\mathrm{OD}_{600}=2.5$. YP medium with $200 \mathrm{~g} / \mathrm{L}$ of lactose containing antibiotics was slowly fed through a pump to maintain the lactose concentration at $10-20 \mathrm{~g} / \mathrm{L}$. The feeding rate was manually adjusted based on lactose consumption and lactose concentration in the fermenter. The culture $\mathrm{pH}$ was automatically maintained at 5.6 by adding $3 \mathrm{~N} \mathrm{NaOH}$ or $\mathrm{HCl}$ solution. The gas flow rate was set at $1 \mathrm{vvm}$, and agitation was set at $800 \mathrm{rpm}$.

Analytical methods and metabolite analysis. The $\mathrm{OD}_{600}$ of cultures was measured using a spectrophotometer (Biomate 5 , Thermo, NY) and dried cell weight (DCW) was obtained from an experimentally determined conversion factor of $0.454 \mathrm{~g} \mathrm{DCW} / \mathrm{OD}_{600}$. Extracellular metabolites such as lactose, galactose, galactitol, glycerol, acetate, and ethanol were measured by HPLC (Agilent Technologies 1200 Series, Santa Clara, CA) with a Rezex ${ }^{\mathrm{TM}}$ ROA-Organic Acid H $+(8 \%)$ column (Phenomenex Inc., Torrance, $\mathrm{CA}$ ) and a refractive index detector (RID). The column was eluted with $0.005 \mathrm{~N} \mathrm{H}_{2} \mathrm{SO}_{4}$ at a flow rate of $0.6 \mathrm{~mL} / \mathrm{min}$ at $50^{\circ} \mathrm{C}$. Galactose and tagatose could not be separated by this ROA-organic acid $\mathrm{H}+(8 \%)$ column (Supplementary Figure 3). Galactose and tagatose concentrations were measured using an Agilent Technologies 1200 Series HPLC equipped with a Rezex ${ }^{\mathrm{TM}}$ RCM-Monosaccharide Ca $+2(8 \%)$ column (Phenomenex Inc. Torrance, $\mathrm{CA}$ ) and RID detector. The mobile phase was E-Pure ${ }^{\mathrm{mm}}$ Water (Barnstead E-pure ${ }^{\mathrm{m}}$ Water Purification Systems, Thermo Scientific) and was eluted at a flow rate of 0.6 $\mathrm{mL} / \mathrm{min}$ at $80^{\circ} \mathrm{C}$. The maxium theoretical tagatose yield $(0.526 \mathrm{~g}$ tagatose $/ \mathrm{g}$ lactose $)$ by engineered yeast was calculated based on the molecular weights of tagatose $(180.16 \mathrm{~g} / \mathrm{mol})$ and lactose $(342.3 \mathrm{~g} / \mathrm{mol})$ with an assumption that glucose is not converted into tagatose.

\section{Data availability}

Data supporting the findings of this work are available within the paper and its Supplementary Information files. A reporting summary for this article is available as a Supplementary Information file. The source data underlying Figs. 1, 2, 3, 4, and 5 are provided as a Source Data file. All other data are available from the corresponding author upon reasonable request.

Received: 29 August 2018 Accepted: 21 February 2019

Published online: 22 March 2019

\section{References}

1. Robinson, P. K., Enzymes: principles and biotechnological applications. Essays Biochem. 59, 1-41 (2015).

2. Zargaraan, A., Kamaliroosta, L., Yaghoubi, A. S. \& Mirmoghtadaie, L. Effect of substitution of sugar by high fructose corn syrup on the physicochemical properties of bakery and dairy products: a review. Nutr. Food Sci. Res. 3, 3-11 (2016).

3. Shin, K. C., Sim, D. H., Seo, M. J. \& Oh, D. K. Increased production of foodgrade D-tagatose from D-galactose by permeabilized and immobilized cells of Corynebacterium glutamicum, a GRAS host, expressing d-galactose isomerase from Geobacillus thermodenitrificans. J. Agric. Food Chem. 64, 8146-8153 (2016).

4. Jayamuthunagai, J., Gautam, P., Srisowmeya, G. \& Chakravarthy, M. Biocatalytic production of D-tagatose: a potential rare sugar with versatile applications. Crit. Rev. Food Sci. Nutr. 57, 3430-3437 (2017).

5. Lim, B. C., Kim, H. J. \& Oh, D. K. High production of D-tagatose by the addition of boric acid. Biotechnol. Prog. 23, 824-828 (2007).

6. Park, C. S. et al. D-allulose production from D-fructose by permeabilized recombinant cells of Corynebacterium glutamicum cells expressing D-allulose 3-epimerase Flavonifractor plautii. PLoS ONE 11, e0160044 (2016).

7. Zhang, W., Yu, S., Zhang, T., Jiang, B. \& Mu, W. Recent advances in D-allulose: physiological functionalities, applications, and biological production. Trends Food Sci. Tech. 54, 127-137 (2016).

8. Staudigl, P., Haltrich, D. \& Peterbauer, C. K. L-Arabinose isomerase and $\mathrm{D}$-xylose isomerase from Lactobacillus reuteri: characterization, coexpression in the food grade host Lactobacillus plantarum, and application in the conversion of D-galactose and D-glucose. J. Agric. Food Chem. 62, 1617-1624 (2014).

9. Chouayekh, H. et al. Characterization of an l-arabinose isomerase from the Lactobacillus plantarum NC8 strain showing pronounced stability at acidic pH. FEMS Microbiol. Lett. 277, 260-267 (2007).

10. Zhang, W. et al. Characterization of a novel metal-dependent D-psicose 3-epimerase from Clostridium scindens 35704. PLoS One 8, e62987 (2013).

11. Kim, H. J., Hyun, E. K., Kim, Y. S., Lee, Y. J. \& Oh, D. K. Characterization of an Agrobacterium tumefaciens D-psicose 3-epimerase that converts D-fructose to D-psicose. Appl. Environ. Microbiol. 72, 981-985 (2006).

12. Hossain, A. et al. Rare sugar D-psicose prevents progression and development of diabetes in T2DM model Otsuka Long-Evans Tokushima Fatty rats. Drug Des. Dev. Ther. 9, 525-535 (2015).

13. Levin, G. V. Tagatose, the new GRAS sweetener and health product. J. Med. Food 5, 23-36 (2002).

14. Lu, Y., Levin, G. V. \& Donner, T. W. Tagatose, a new antidiabetic and obesity control drug. Diabetes Obes. Metab. 10, 109-134 (2008).

15. Kim, P. Current studies on biological tagatose production using D-arabinose isomerase: a review and future perspective. Appl. Microbiol. Biotechnol. 65, 243-249 (2004).

16. Leang, K. et al. Novel reactions of L-rhamnose isomerase from Pseudomonas stutzeri and its relation with $\mathrm{D}$-xylose isomerase via substrate specificity. Biochim. Et. Biophys. Acta (BBA)-General Subj. 1674, 68-77 (2004).

17. Leang, K. et al. A novel enzymatic approach to the massproduction of L-galactose from L-sorbose. J. Biosci. Bioeng. 97, 383-388 (2004).

18. Wanarska, M. \& Kur, J. A method for the production of D-tagatose using a recombinant Pichia pastoris strain secreting beta-D-galactosidase from Arthrobacter chlorophenolicus and a recombinant L-arabinose isomerase from Arthrobacter sp. 22c. Microb. Cell. Fact. 11, 113 (2012).

19. Liu, J. J. et al. Lactose fermentation by engineered Saccharomyces cerevisiae capable of fermenting cellobiose. J. Biotechnol. 234, 99-104 (2016).

20. Seiboth, B., Gamauf, C., Pail, M., Hartl, L. \& Kubicek, C. P. The D-xylose reductase of Hypocrea jecorina is the major aldose reductase in pentose and D-galactose catabolism and necessary for beta-galactosidase and cellulase induction by lactose. Mol. Microbiol. 66, 890-900 (2007).

21. Jagtap, S. S., Singh, R., Kang, Y. C., Zhao, H. \& Lee, J. K. Cloning and characterization of a galactitol 2-dehydrogenase from Rhizobium legumenosarum and its application in D-tagatose production. Enzym. Microb. Technol. 58-59, 44-51 (2014)

22. Oh, E. J. et al. Gene amplification on demand accelerates cellobiose utilization in engineered Saccharomyces cerevisiae. Appl. Environ. Microbiol. 82, 3631-3639 (2016) 
23. De Robichon-Szulmajster, H. Induction of enzymes of the galactose pathway in mutants of Saccharomyces cerevisiae. Science 127, 28-29 (1958).

24. DiCarlo, J. E. et al. Genome engineering in Saccharomyces cerevisiae using CRISPR-Cas systems. Nucleic Acids Res. 41, 4336-4343 (2013).

25. Zhang, G. C. et al. Construction of a quadruple auxotrophic mutant of an industrial polyploid saccharomyces cerevisiae strain by using RNA-guided Cas9 nuclease. Appl. Environ. Microbiol. 80, 7694-7701 (2014).

26. Cheng, L., Mu, W. \& Jiang, B. Thermostable L-arabinose isomerase from Bacillus stearothermophilus IAM 11001 for D-tagatose production: gene cloning, purification and characterisation. J. Sci. Food Agric. 90, 1327-1333 (2010).

27. Kim, B.-C. et al. Cloning, expression and characterization of L-arabinose isomerase from Thermotoga neapolitana: bioconversion of $\mathrm{D}$-galactose to $\mathrm{D}$ tagatose using the enzyme. FEMS Microbiol. Let. 212, 121-126 (2002).

28. Nguyen, T. K., Hong, M. G., Chang, P. S., Lee, B. H. \& Yoo, S. H. Biochemical properties of $\mathrm{L}$-arabinose isomerase from Clostridium hylemonae to produce D-tagatose as a functional sweetener. PLoS ONE 13, e0196099 (2018).

29. Li, X. et al. Comparison of xylose fermentation by two high-performance engineered strains of Saccharomyces cerevisiae. Biotechnol. Rep. (Amst.) 9 , 53-56 (2016).

30. Mitsuhashi, S. \& Lampen, J. O. Conversion of D-xylose to D-xylulose in extracts of Lactobacillus pentosus. J. Biol. Chem. 204, 1011-1018 (1953).

31. Lee, C. Y., Bagdasarian, M., Meng, M. H. \& Zeikus, J. G. Catalytic mechanism of xylose (glucose) isomerase from Clostridium thermosulfurogenes. Characterization of the structural gene and function of active site histidine. J. Biol. Chem. 265, 19082-19090 (1990).

32. Wei, N., Xu, H., Kim, S. R. \& Jin, Y. S. Deletion of FPS1, encoding aquaglyceroporin Fpslp, improves xylose fermentation by engineered Saccharomyces cerevisiae. Appl. Environ. Microbiol. 79, 3193-3201 (2013).

33. Zhan, Y. et al. Coexpression of beta-D-galactosidase and L-arabinose isomerase in the production of D-tagatose: a functional sweetener. J. Agric. Food Chem. 62, 2412-2417 (2014).

34. Ha, S. J. et al. Engineered Saccharomyces cerevisiae capable of simultaneous cellobiose and xylose fermentation. Proc. Natl Acad. Sci. USA 108, 504-509 (2011).

35. Zhang, G. C., Liu, J. J., Kong, I. I., Kwak, S. \& Jin, Y. S. Combining C6 and C5 sugar metabolism for enhancing microbial bioconversion. Curr. Opin. Chem. Biol. 29, 49-57 (2015)

36. Liu, J. J. et al. Metabolic engineering of probiotic Saccharomyces boulardii. Appl. Environ. Microbiol. 82, 2280-2287 (2016).

37. Jin, Y. S. \& Jeffries, T. W. Changing flux of xylose metabolites by altering expression of xylose reductase and xylitol dehydrogenase in recombinant Saccharomyces cerevisiae. Appl. Biochem. Biotechnol. 105 -108, 277-286 (2003).

38. Mumberg, D., Muller, R. \& Funk, M. Yeast vectors for the controlled expression of heterologous proteins in different genetic backgrounds. Gene 156, 119-122 (1995).

39. Taxis, C. \& Knop, M. System of centromeric, episomal, and integrative vectors based on drug resistance markers for Saccharomyces cerevisiae. Biotechniques 40, 73-78 (2006).
40. Kwak, S. et al. Enhanced isoprenoid production from xylose by engineered Saccharomyces cerevisiae. Biotechnol. Bioeng. 114, 2581-2591 (2017).

\section{Acknowledgements}

This work was funded by Energy Biosciences Institute and the DOE Center for Advanced Bioenergy and Bioproducts Innovation (U.S. Department of Energy, Office of Science, Office of Biological and Environmental Research under Award Number DE-SC0018420). We thank Christine Anne Atkinson and Cassie Liu for proofreading the paper.

\section{Author contributions}

Y.-S.J., J.-J.L. and G.-C.Z. designed research. J.-J.L. and G.-C.Z. performed research and analyzed data. S.K. and E.J.O. helped with bioreactor fermentation. J.-J.L., G.-C.Z., E.J.Y., K.C., J. H.D.C., and Y.-S.J. wrote the paper. All authors read and approved the paper.

\section{Additional information}

Supplementary Information accompanies this paper at https://doi.org/10.1038/s41467019-09288-6.

Competing interests: J.-J.L., G.-C.Z., K.C., J.H.D.C., and Y.-S.J. are authors of a patent application filed by University of Illinois at Urbana-Champaign based on this work. K.C. J.H.D.C. and Y.-S.J. have financial interest in Sugarlogix, Inc. The remaining authors declare no competing interests.

Reprints and permission information is available online at http://npg.nature.com/ reprintsandpermissions/

Journal Peer Review Information: Nature Communications thanks Deok-Kun Oh, Michael Naesby, and other anonymous reviewer(s) for their contribution to the peer review of this work. Peer reviewer reports are available.

Publisher's note: Springer Nature remains neutral with regard to jurisdictional claims in published maps and institutional affiliations.

cc) (i) Open Access This article is licensed under a Creative Commons Attribution 4.0 International License, which permits use, sharing, adaptation, distribution and reproduction in any medium or format, as long as you give appropriate credit to the original author(s) and the source, provide a link to the Creative Commons license, and indicate if changes were made. The images or other third party material in this article are included in the article's Creative Commons license, unless indicated otherwise in a credit line to the material. If material is not included in the article's Creative Commons license and your intended use is not permitted by statutory regulation or exceeds the permitted use, you will need to obtain permission directly from the copyright holder. To view a copy of this license, visit http://creativecommons.org/ licenses/by/4.0/.

(C) The Author(s) 2019 